\title{
Prevalence of Diabetes and Its Relationship With Body Mass Index Among Elderly People in a Rural Area of Northeastern State of India
}

\author{
Gajendra K. Medhi ${ }^{1}$, Gitashree Dutta ${ }^{1}$, Prasanta Borah ${ }^{2}$, Markordor Lyngdoh ${ }^{1}$, Amitav Sarma ${ }^{3}$ \\ 1. Community Medicine, North Eastern Indira Gandhi Regional Institute of Health \& Medical Sciences, Shillong, IND 2. \\ Epidemiology and Nutrition, ICMR-Regional Medical Research Centre, Northeast (NE) Region, Dibrugarh, IND 3. \\ Anatomy, North Eastern Indira Gandhi Regional Institute of Health \& Medical Sciences, Shillong, IND
}

Corresponding author: Gajendra K. Medhi, gkmedhi@hotmail.com

\section{Abstract}

\section{Background}

Diabetes and its complications are a major public health concern in elderly populations. However, there is little population-based data on diabetes and its risk factors among the elderly population living in rural areas of India. The objective of this population-based study was to assess the prevalence of diabetes in the elderly population and its relationship with body mass index (BMI).

\section{Methodology}

A population-based, cross-sectional study was conducted among elderly individuals ( $\geqslant 60$ years) during the period 2013-2016 in rural areas of Dibrugarh district of Assam. Multi-stage sampling design was adopted to select the study participants. Data on socio-demographic profile and diagnosis/treatment history of diabetes were collected using pre-designed and pre-tested questionnaire. Fasting blood sugar was tested. Weight and height were measured to calculate BMI. Multivariate logistic regression analysis was performed to assess the relationship between diabetes and BMI.

\section{Results}

Data were collected from 430 (male: 210 , female: 220 ) individuals. The overall prevalence of diabetes was 7.9\% (male: $7.1 \%$, female: 8.6\%). Higher level of education was associated with increased prevalence of diabetes. Prevalence of diabetes increased as the BMI of participants increased. Prevalence of diabetes among obese individuals ( $\mathrm{BMI} \geqslant 25 \mathrm{~kg} / \mathrm{m}^{2}$ ) was $30.4 \%$ compared to only $5 \%$ among normal weight individuals. Obesity was associated with eight-fold higher risk of diabetes compared with the individuals of normal weight in multivariate analysis.

\section{Conclusions}

Review began 01/10/2021 Review ended 01/13/2021 Published 01/17/2021

() Copyright 2021

Medhi et al. This is an open access article distributed under the terms of the Creative Commons Attribution License CC-BY 4.0., which permits unrestricted use, distribution, and reproduction in any medium, provided the original author and source are credited.
The study reveals nearly $8 \%$ population-based prevalence of diabetes in rural elderly people in the study district. Our study provides epidemiological evidence that obesity is a major driver of diabetes among rural elderly people.

Categories: Endocrinology/Diabetes/Metabolism, Public Health, Epidemiology/Public Health Keywords: elderly, diabetes, bmi, obesity

\section{Introduction}

Diabetes mellitus has steadily evolved as a major public health problem over the past quarter century in India and across the globe [1]. India is contributing a major part of the global diabetes burden, and is considered the diabetic capital of the world [1,2]. India has an estimated 77 million people with diabetes, which is the second highest in the world next to China. Diabetes burden is projected to be 101 million in 2030 and 134 million in 2045 [3]. Population ageing is attributed to be one of the driving forces behind the increase in diabetes prevalence in India [4]. Earlier studies have reported that the prevalence of diabetes mellitus is higher among the elderly compared with middle-aged or young adults [5-7].

Historically a disease of the affluent, recent epidemiological evidence indicates a rising diabetes mellitus incidence and prevalence in urban India's middle-class and working-class poor sections as well as among the rural populations $[6,8]$. Studies from different parts of India have provided evidence of the rising prevalence of overweight/obesity in India, and overweight/obesity have been found to be most important contributor of rising prevalence of diabetes in the country $[4,6]$.

Diabetes and its complications may take a major toll on the quality of life of the elderly [9]. Diabetes in the 
elderly can cause substantial morbidity from macro- and micro-vascular complications, higher mortality, reduced functional status, and increased risk of institutionalization [9-12]. Hence, it is imperative to understand the burden and risk factors of diabetes in the elderly population. However, there is a paucity of population-based data on the prevalence and risk factors of diabetes among the rural elderly population where more than $70 \%$ of the population resides, especially in the northeastern parts of India [8]. This study aimed to investigate the prevalence of diabetes among the elderly population in a rural area of a northeastern state of India along with its relationship with body mass index (BMI).

\section{Materials And Methods}

A community-based, cross-sectional study was conducted during the period 2013-2016 among elderly individuals aged $\geqslant 60$ years in rural areas of Dibrugarh district of Assam, India. A multi-stage sampling design was adopted to recruit the participants into the study. In the first stage, two development blocks out of seven were selected randomly from the total list of development blocks in the district. In the last stage, seven villages from each selected blocks were chosen to conduct the study. All community-dwelling individuals 60 years and above were eligible to participate in the study. A total of 430 eligible individuals were recruited in the study. The study protocol was reviewed and approved by the Institutional Ethical Committee of Regional Medical Research Centre (RMRC), Dibrugarh. Informed consent was taken from all the respondents before data collection.

After obtaining informed consent, all the participants were interviewed by trained interviewers using a predesigned and pre-tested questionnaire through face-to-face interview to collect data on socio-demographic variables and treatment seeking behaviors.

All individuals who were diagnosed as diabetic by a physician, and/or under treatment for diabetes (i.e., insulin and/or oral hypoglycemic agents), and/or individuals who had a fasting blood glucose level of $\geqslant 126$ $\mathrm{mg} / \mathrm{dL}$ after an overnight fasting of at least eight hours, and/or two-hour post-glucose value of $\geqslant 200 \mathrm{mg} / \mathrm{dL}$ were considered to have diabetes $[13,14]$. Capillary blood glucose (CBG) was measured using a standardized digital glucometer (Accu-Check, Roche diagnostics, Indianapolis, IN, USA) [14]. The blood collection and testing on the spot was carried out as per the guidelines provided in the manual of the company. Blood glucose level in $\mathrm{mg} / \mathrm{dL}$ was recorded from the monitor.

Measurements of height and weight were done as per the guidelines of the World Health Organization (WHO) $[14,15]$. The weight was recorded to a minimum of $0.5 \mathrm{~kg}$. Similarly, the height was recorded to a minimum of $0.5 \mathrm{~cm}$.

BMI of participants was calculated using the formula: weight $(\mathrm{kg}) /$ height $\left(\mathrm{m}^{2}\right)$. Participants were divided into four groups for both males and females according to the WHO Asia Pacific guidelines: Underweight: BMI $<18.5 \mathrm{~kg} / \mathrm{m}^{2}$; normal weight: BMI 18.5-22.99 kg/m²; overweight: BMI 23-24.99 kg/m²; and obese: BMI $\geqslant 25$ $\mathrm{kg} / \mathrm{m}^{2}[16]$.

\section{Statistical analysis}

Data were entered and analyzed using SPSS version 21 (IBM, Armonk, NY, USA). Descriptive statistics such as frequency and mean with standard deviation (SD) were used to present the results. Chi-square test was used for comparison of proportions across groups, and t-test was used to compare continuous variables. Univariate and multivariate binary logistic regression analyses were carried out to produce crude and adjusted odds ratios (ORs) with $95 \%$ confidence intervals to examine the relationships between outcome variable (i.e., diabetes) and explanatory variables (i.e., BMI) after adjustment for socio-demographic variables such as age, gender, educational status, and marital status. A p-value of $<0.05$ was considered statistically significant for all the statistical procedures.

\section{Results}

Table 1 shows the socio-demographic characteristics of the study participants. A total of 430 (male: 210, female: 220) individuals participated in the study. The mean age of the participants was 68.71 (SD: 7.42) years. Most of the participants were in the age group of 60-69 (58.1\%) years, $53.4 \%$ were currently married (58.6\%), and $30.5 \%$ had no formal education. Overall, $14.1 \%$ of the participants were obese. The overall prevalence of diabetes was $7.9 \%(n=34)$ among the study participants. 


\section{Cureus}

\begin{tabular}{|c|c|c|}
\hline \multicolumn{2}{|l|}{ Variables } & n (\%) \\
\hline \multirow{4}{*}{ Age (years) } & $60-69$ & $250(58.1)$ \\
\hline & $70-79$ & $138(32.1)$ \\
\hline & $80+$ & $42(9.8)$ \\
\hline & Men age \pm SD & $68.71 \pm 7.42$ \\
\hline \multirow{2}{*}{ Gender } & Male & $210(48.8)$ \\
\hline & Female & $220(51.2)$ \\
\hline \multirow{3}{*}{ Educational status } & No formal education & $131(30.5)$ \\
\hline & Up to high school & $224(52.1)$ \\
\hline & Beyond high school & 75 (17.4) \\
\hline \multirow{4}{*}{ Marital status } & Married & $252(58.6)$ \\
\hline & Widowed & $173(40.2)$ \\
\hline & Separated/divorced & $2(0.5)$ \\
\hline & Unmarried & $3(0.7)$ \\
\hline \multirow{4}{*}{ BMI $\left(\mathrm{kg} / \mathrm{m}^{2}\right) \quad(\mathrm{N}=399)$} & Underweight & $127(32)$ \\
\hline & Normal weight & $161(40.6)$ \\
\hline & Overweight & $53(13.4)$ \\
\hline & Obese & $56(14.1)$ \\
\hline \multirow{2}{*}{ Diabet } & Present & $34(7.9)$ \\
\hline & Absent & 396 (92.1) \\
\hline Total & & 430 \\
\hline
\end{tabular}

TABLE 1: Characteristics of study participants.

SD, standard deviation; BMI, body mass index

Among the 34 diabetic participants, 27 were known diabetics and seven were newly diagnosed during the survey.

Table 2 shows the prevalence of diabetes stratified by age, gender, educational, and marital status. No significant gender difference was found in the prevalence. Age trend indicates that diabetes was confined mostly in the age group of 60-69 years, and none of the participants were found to have diabetes in the $>80$ year age group. The highest educational group (i.e., beyond high school level) had the highest prevalence compared to their less educated counterparts. Married participants had significantly higher prevalence of diabetes than widowed. 


\section{Cureus}

\begin{tabular}{|c|c|c|}
\hline \multirow{2}{*}{ Variables } & Prevalence & \multirow{2}{*}{ P-Value } \\
\hline & $n(\%)$ & \\
\hline \multicolumn{2}{|l|}{ Age (years) } & \multirow{4}{*}{0.007} \\
\hline $60-69(n=250)$ & $25(10)$ & \\
\hline $70-79(n=138)$ & $9(6.5)$ & \\
\hline $80+(n=42)$ & $0(0)$ & \\
\hline \multicolumn{2}{|l|}{ Gender } & \multirow{3}{*}{0.347} \\
\hline Male $(n=210)$ & $15(7.1)$ & \\
\hline Female $(n=220)$ & $19(8.6)$ & \\
\hline \multicolumn{2}{|l|}{ Educational status } & \multirow{4}{*}{0.009} \\
\hline No formal education $(n=131)$ & $8(4.3)$ & \\
\hline Less than high school $(n=224)$ & $3(4.5)$ & \\
\hline Beyond high school $(\mathrm{n}=75)$ & $23(13)$ & \\
\hline \multicolumn{2}{|l|}{ Marital status } & \multirow{3}{*}{0.05} \\
\hline Married $(n=252)$ & $25(9.9)$ & \\
\hline Widowed $(n=173)$ & $9(5.2)$ & \\
\hline
\end{tabular}

\section{TABLE 2: Prevalence of diabetes according to socio-demographic variables.}

Table 3 presents the mean BMI level according to the diabetes status of the participants. The mean BMI was found to be significantly higher in diabetics than non-diabetics in both males and females.

\begin{tabular}{|c|c|c|c|}
\hline & \multicolumn{2}{|c|}{ Mean BMI \pm SD } & \multirow{2}{*}{ P-Value } \\
\hline & Diabetics & Non-diabetics & \\
\hline Male & $23.96 \pm 4.62$ & $19.97 \pm 3.82$ & 0.000 \\
\hline Female & $25.35 \pm 4.34$ & $20.69 \pm 5.1$ & 0.000 \\
\hline Total & $24.75 \pm 4.45$ & $20.33 \pm 4.51$ & 0.000 \\
\hline
\end{tabular}

\section{TABLE 3: Mean BMI (diabetics versus non-diabetics).}

BMI, body mass index; SD, standard deviation

Table 4 shows that the prevalence of diabetes increased as the BMI level of participants increased. Participants with the lowest BMI (i.e., underweight individuals with BMI of $<18.5 \mathrm{~kg} / \mathrm{m}^{2}$ ) had the lowest prevalence (2.4\%) and those with the highest BMI (i.e., obese individuals with BMI of $\geqslant 25 \mathrm{~kg} / \mathrm{m}^{2}$ ) had the highest prevalence of diabetes (30.4\%). 


\section{Cureus}

\begin{tabular}{|l|l|}
\hline BMI categories & Prevalence, $\mathbf{n}(\%)$ \\
\hline Underweight & $3(2.4)$ \\
Normal weight & $8(5)$ \\
Overweight & $4(7.5)$ \\
Obese & $17(30.4)$ \\
P-value & $<0.0001$ \\
\hline
\end{tabular}

TABLE 4: Association between diabetes and BMI.

BMI, body mass index

Table 5 presents the results of univariate and multivariate logistic regression analyses examining the relationship between diabetes and BMI. In the univariate logistic regression analysis, the crude ORs increased with increasing BMI level. A similar pattern was also observed in multiple logistic regression analysis. The adjusted ORs showed that obese individuals (the highest BMI category) had 8.13 times higher risk of having diabetes compared with normal-weight individuals. Although not statistically significant, being overweight was also associated with nearly 1.5 -fold higher odds of having diabetes compared with normal-weight individuals.

\begin{tabular}{|c|c|c|}
\hline BMI categories & COR (95\% Cl) & AOR (95\% Cl) \\
\hline Underweight & $0.463(0.120-1.78)$ & $0.523(0.132-2.068)$ \\
\hline Normal weight & 1 (reference) & 1 (reference) \\
\hline Overweight & $1.56(0.451-5.41)$ & $1.52(0.425-5.4)$ \\
\hline Obese & $8.34(3.35-20.73)^{\star \star}$ & $8.13(3.19-20.7)^{\star \star}$ \\
\hline
\end{tabular}

\section{TABLE 5: Univariate and multivariate logistic regression analyses showing association between diabetes and BMI.}

$\mathrm{BMI}$, body mass index; COR, crude odds ratio; AOR, adjusted odds ratio

** Significant $\mathrm{P}$-value $(\mathrm{P}<0.01)$

\section{Discussion}

This population-based, cross-sectional study documents the prevalence of diabetes among the elderly population (>60 years) in a northeastern state of India, and reveals strong epidemiological evidence of association between BMI and diabetes in this population. The overall prevalence of diabetes was $7.9 \%$ in this study. There are no population-based data on the prevalence of diabetes among the elderly in Assam for comparison; however, the prevalence documented in this study was higher than the overall prevalence of $4.4 \%$ among adult population (>20 years) in Assam found in a recent population-based study [9]. The present prevalence is slightly lower than the national prevalence of about $10 \%$ among the rural elderly (>65 years) [9]. However, the prevalence was found to be much lower compared to $23 \%$ reported by Medhi et al. among the urban elderly in a recent population-based study conducted in the same district, which reflects existing rural-urban differences in the prevalence of diabetes in India [17]. Our study adds to the limited but growing body of evidence suggesting that diabetes is no longer confined to the urban areas of India but is gradually emerging as a public health concern in rural areas as well $[6,8,18]$. Considering that over $70 \%$ of India's population resides in rural areas, even a small increase in the prevalence of diabetes in rural areas will imply that a large proportion of individuals will require chronic care as rural populations already suffer with issues such as poor access to health services and poverty $[6,8]$.

Out of 34 total diabetics, 27 were known diabetics and seven (20.5\%) were newly diagnosed during the survey, indicating a significant burden of undiagnosed diabetes in the elderly. In an earlier study conducted in Assam, prevalence of undiagnosed diabetes was found to be $50 \%$ among adult rural population ( $>20$ years) [6]. Incidental diagnosis of diabetes is generally more common in elderly individuals because of their 
frequent healthcare contact; hence, prevalence of undiagnosed diabetes is likely to be less in the elderly than younger populations $[6,19]$.

Age-specific prevalence indicates that diabetes was more common among the 60-69-year age group, and none were found to have diabetes in the $>80$-year age group. Higher prevalence in younger age group probably reflects that diabetes among the elderly in rural population is a relatively recent phenomenon. In this study, no gender difference in the prevalence of diabetes was revealed, which is consistent with findings from other Indian studies [7,8,17,20,21]. However, few studies have documented male preponderance of diabetes [8].

The highest educational group had the highest prevalence of diabetes in this study, which confirms the earlier report from a large multicenter study $[5,6]$. Such findings suggest that diabetes remains a disease of the more affluent sections of society in the rural areas of India [6]. Higher prevalence in currently married elderly compared with widowed could be due to the younger age of married participants.

It is clear from this study that obesity is an important risk factor of diabetes in this population, which confirms the findings of earlier studies from India [5,6,8,18,20-22]. We found that the prevalence of diabetes increased with increasing BMI level as the prevalence of diabetes was only $2.4 \%$ among underweight individuals and 5\% among normal-weight individuals which increased to $7.5 \%$ among overweight and $30.4 \%$ among obese individuals. In multivariate analysis, obesity was associated with more than a 8.3-fold higher risk of diabetes compared with normal-weight individuals. Although not statistically significant, being overweight was also associated with a 1.5-fold higher risk of diabetes compared with normal-weight individuals. Obesity was found to be a strong risk factor in this study, even though we used a lower BMI cutoff value $\left(B M I \geqslant 25 \mathrm{~kg} / \mathrm{m}^{2}\right.$ ) to define obesity suggested for Asian populations as opposed to the standard cutoff value (BMI $\geqslant 30 \mathrm{~kg} / \mathrm{m}^{2}$ ). In general, Indians have a lower BMI than those of Europeans; however, the risk of diabetes increases at very low levels of BMI for Indians $[8,16]$. As the obesity epidemic is showing an increasing trend in India, there may be further escalation of obesity-related diabetes in this population, warranting greater public efforts to contain the obesity epidemic [23].

\section{Limitations}

The study had few limitations. First, as the study is a cross-sectional study, it is difficult to draw causal inferences. Second, various important confounding variables (such as family history of diabetes, dietary, and life-style factors) were not taken into account in the multivariate analysis, which may limit us in elucidating the true relationship between explanatory variables (i.e., BMI) and outcome variables (i.e., diabetes). Third, measurement of CBG was done by a glucometer device instead of venous blood glucose estimation due to logistical issues. Several previous studies have reported that CBG is a feasible alternative for screening in epidemiological studies in which obtaining venous samples might be difficult. Finally, the study was conducted in one district; hence, the findings of the study are not generalizable to the entire region or country.

\section{Conclusions}

The present study provides reliable and recent epidemiological data on the prevalence of diabetes mellitus among the rural elderly population in a representative population in a district of Assam, India. Approximately $8 \%$ of the general elderly rural population have diabetes mellitus, indicating that diabetes is emerging as an important public health issue in the rural areas of India, warranting comprehensive public health actions for prevention, screening/diagnosis, and ensuring accessibility to treatment and care to the growing number of diabetics in rural areas. This study also highlights that obesity is a key parameter associated with diabetes mellitus even in the rural populations in India. Given the current increasing trend of obesity in the country, prevalence of obesity-associated diabetes is expected to further increase in the rural population. Weight control programs, particularly among the younger populations, will be costeffective in addressing the diabetes epidemic. For more effective public health actions, further detailed studies should be conducted to investigate the risk factors of diabetes and obesity among rural populations in India.

\section{Additional Information \\ Disclosures}

Human subjects: Consent was obtained or waived by all participants in this study. Regional Medical Research Centre, N.E. Region, Dibrugarh issued approval N0.RMRC/Dib/IEC(Human)2012-13/2220, dated 19.10.2012. Recommended for carrying out the study. Animal subjects: All authors have confirmed that this study did not involve animal subjects or tissue. Conflicts of interest: In compliance with the ICMJE uniform disclosure form, all authors declare the following: Payment/services info: Received an extramural grant to carry out this study. Financial relationships: All authors have declared that they have no financial relationships at present or within the previous three years with any organizations that might have an interest in the submitted work. Other relationships: All authors have declared that there are no other relationships or activities that could appear to have influenced the submitted work. 


\section{Acknowledgements}

The authors are grateful to the Indian Council of Medical Research (ICMR), New Delhi for financial support to carry out this study.

\section{References}

1. India State-Level Disease Burden Initiative Diabetes Collaborators: The increasing burden of diabetes and variations among the states of India: the Global Burden of Disease Study 1990-2016. Lancet Glob Health. 2018, 6:1352-62. 10.1016/S2214-109X(18)30387-5

2. Joshi SR, Parikh RM: India--diabetes capital of the world: now heading towards hypertension . J Assoc Physicians India. 2007, 55:323-42.

3. Saeedi P, Petersohn I, Salpea P, et al.: Global and regional diabetes prevalence estimates for 2019 and projections for 2030 and 2045: results from the International Diabetes Federation Diabetes Atlas, 9th edition. Diabetes Res Clin Pract. 2019, 157:107843. 10.1016/j.diabres.2019.107843

4. Kutty VR, Dilip TR, Archana AR, Gopinathan S, Ramanathan M: Shifting pattern of diabetes among the elderly in India: evidence from the national sample survey organization's data, 2004-2014. Int J NonCommun Dis. 2018, 3:67-74. 10.4103/jncd.jncd_37_17

5. Kapil U, Khandelwal R, Ramakrishnan L, et al.: Prevalence of hypertension, diabetes, and associated risk factors among geriatric population living in a high-altitude region of rural Uttarakhand, India. J Family Med Prim Care. 2018, 7:1527-36. 10.4103/jfmpc.jfmpc_108_18

6. Anjana RM, Deepa M, Pradeepa R, et al.: Prevalence of diabetes and prediabetes in 15 states of India: results from the ICMR-INDIAB population-based cross-sectional study. Lancet Diabetes Endocrinol. 2017, 5:58596. 10.1016/S2213-8587(17)30174-2

7. Goswami AK, Gupta SK, Kalaivani M, Nongkynrih B, Pandav CS: Burden of hypertension and diabetes among urban population aged $\geqslant 60$ years in South Delhi: a community based study. J Clin Diagn Res. 2016, 10:1-5. 10.7860/JCDR/2016/17284.7366

8. Tripathy JP, Thakur JS, Jeet G, et al.: Prevalence and risk factors of diabetes in a large community-based study in North India: results from a STEPS survey in Punjab, India. Diabetol Metab Syndr. 2017, 9:8. 10.1186/s13098-017-0207-3

9. Jain A, Paranjape S: Prevalence of type 2 diabetes mellitus in elderly in a primary care facility: an ideal facility. Indian J Endocrinol Metab. 2013, 17:318-22. 10.4103/2230-8210.119647

10. Kirkman MS, Briscoe VJ, Clark N, et al.: Diabetes in older adults. Diabetes Care. 2012, 35:2650-64. 10.2337/dc12-1801

11. Brown AF, Mangione CM, Saliba D, Sarkisian CA, California Healthcare Foundation/American Geriatrics Society Panel on Improving Care for Elders with Diabetes: Guidelines for improving the care of the older person with diabetes mellitus. J Am Geriatr Soc. 2003, 51:265-80. 10.1046/j.1532-5415.51.5s.1.x

12. Meneilly GS, Tessier D: Diabetes in elderly adults . J Gerontol A Biol Sci Med Sci. 2001, 56:5-13. 10.1093/gerona/56.1.m5

13. American Diabetes Association: Classification and diagnosis of diabetes. Diabetes Care. 2015, 38:8-16. 10.2337/dc15-S005

14. WHO STEPS surveillance manual. Geneva, Switzerland: World Health Organization . (2017). Accessed: January 4, 2021: https://www.who.int/ncds/surveillance/steps/STEPS_Manual.pdf.

15. Physical status: the use and interpretation of anthropometry. Technical report Series No 854. Geneva; World Health Organization. (1995). Accessed: January 4, 2021: https://apps.who.int/iris/bitstream/handle/10665/37003/WHO_TRS_854.pdf?sequence=1.

16. The Asia Pacific perspective: redefining obesity and its treatment . (2000). Accessed: December 24, 2020: https://apps.who.int/iris/handle/10665/206936.

17. Medhi GK, Sarma J: Self-rated health (SRH) among elderly diabetics in an urban setting of Assam, India . Int J Health Sci Res. 2015, 5:253-9.

18. Little M, Humphries S, Patel K, Dodd W, Dewey C: Factors associated with glucose tolerance, pre-diabetes, and type 2 diabetes in a rural community of South India: a cross-sectional study. Diabetol Metab Syndr. 2016, 8:21. 10.1186/s13098-016-0135-7

19. Sinclair A, Dunning T, Rodriguez-Mañas L: Diabetes in older people: new insights and remaining challenges. Lancet Diabetes Endocrinol. 2015, 3:275-85. 10.1016/S2213-8587(14)70176-7

20. Ramachandran A, Snehalatha C, Kapur A, et al.: High prevalence of diabetes and impaired glucose tolerance in India: National Urban Diabetes Survey. Diabetologia. 2001, 44:1094-101. 10.1007/s001250100627

21. Barik A, Mazumdar S, Chowdhury A, Rai RK: Physiological and behavioral risk factors of type 2 diabetes mellitus in rural India. BMJ Open Diabetes Res Care. 2016, 4:255. 10.1136/bmjdrc-2016-000255

22. Vasanthakumar J, Kambar S: Prevalence of obesity among type 2 diabetes mellitus patients in urban areas of Belagavi. Indian J Health Sci Biomed Res. 2020, 13:21-7. 10.4103/kleuhsj.kleuhsj_221_18

23. Luhar S, Timæus IM, Jones R, et al.: Forecasting the prevalence of overweight and obesity in India to 2040 . PLoS One. 2020, 15:229438. 10.1371/journal.pone.0229438 